\title{
Aspects in Topology-Based Geometric Modeling Possible Tools for Discrete Geometry?
}

\author{
Pascal Lienhardt \\ Université de Poitiers, IRCOM-SIC (UMR CNRS 6615) \\ SP2MI, Bvd. 3, Téléport 2 \\ F-86960 Futuroscope Cedex, France
}

\begin{abstract}
Topology-based Geometric Modeling is concerned with modeling subdivisions of geometric spaces. Methods are close to that of combinatorial topology, but for different purposes. We discuss some of these methods, their interests and drawbacks for Geometric Modeling, mainly aspects we think that could be of possible interest for Discrete Geometry.
\end{abstract}

\section{Introduction}

Geometric objects handled in Geometric Modeling are often structured point sets, since it is important for many applications, not only to represent a geometric object, but also to distinguish between different parts of the object, according to properties which are relevant for the application (e.g. mechanical, photometric, geometric properties). Important consequences exist for interactive or procedural modelers; for instance, the structure of a modelled object has to be taken into account when constructing it (it can be used for controlling construction operations).

Topology-based Geometric Modeling deals with subdivisions of geometric spaces, i.e. partitions of these spaces into cells: vertices, edges, faces, volumes, etc. which define a sort of discretization of the geometric space. The fundamentals of many approaches are close to that of Combinatorial Topology [Ale] [SeTh] [FrPi], but for different purposes, and consist in distinguishing between topological information and embedding information. Topological information can be captured by combinatorial structures and controlled by combinatorial operations. So implementation of these structures (and related algorithms) can be done without loss of information and without loss of properties.

More precisely, a subdivision is represented using a combinatorial structure which describes its topology (mainly the cells and their boundary relations), and embedding information is associated with topological information in order to describe the location of the subdivision into its embedding space (usually $E^{2}$ or $E^{3}$ ). Different methods exist for defining embedding: linear embedding, free-form spaces, hierarchized embedding (a subdivision is embedded onto an other subdivision, in order to distinguish between different representation levels, for instance).

An important consequence of this distinction between topology and embedding is that it is necessary to maintain a strong consistency between these two 
complementary aspects, and this has to be taken into account when designing construction operations [Tak].

Nevertheless, a main interest of topology-based geometric modeling is the fact that the relations between combinatorial structures and subdivisions of geometric spaces have been extensively studied by mathematicians. So, a lot of knowledge can be directly used: for instance, many results in combinatorial topology can be used for computing properties, which are useful for controlling (constructions of) modeled objects. Such controls are essential for most applications. Moreover, it is possible to modify several geometric algorithms (e.g. for rendering, finite element method), which classically perform numerical computations, so that they will exploit topological information, and perform combinatorial computations, increasing thus their reliability and efficiency.

Different types of subdivisions have to be handled, according to the applications: general complexes or particular manifolds, subdivided into any cells or regular ones (e.g. simplices): cf. Figure 1. Since 25 years, numerous structures, operations, modelers have been conceived in topology-based geometric modeling: first, cellular subdivisions of 2-dimensional manifolds are studied for Boundary Representation of solids [AFF] [Bau] [Bra] [Män] [Wei1], but recent works deal with simplicial [FePa] [LaLi1] [PBCF] or cellular geometric complexes [CCM] [CrRe] [ElLi1] [GCP] [LuLu] [Mar] [MuHi] [RoOC] [Wei2] or manifolds [ArKo] [DoLa] [GuSt] [HC] [Lie1] [Spe].

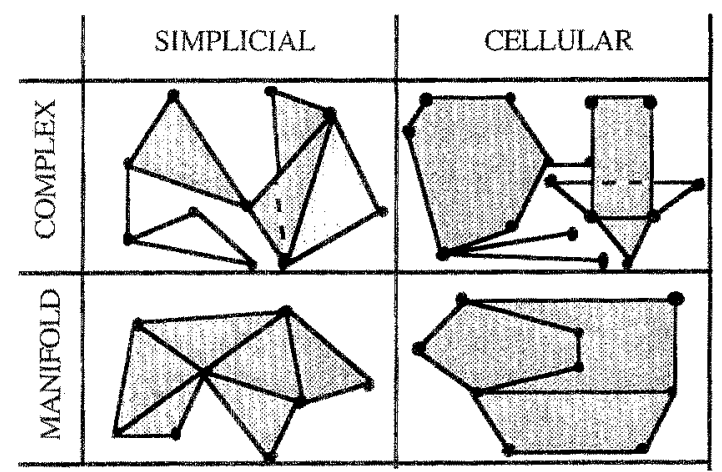

Fig. 1. Several types of topologically 2-dimensional subdivisions. Intuitively, complexes are any assemblies of cells (cells are regular, as simplices, or not). The neighborhood of any point of an n-dimensional manifolds is an $n$-ball (or half ball if the point lies on a boundary).

In order to illustrate previous remarks and assertions, we will focus on several works whose fundamentals are well-known in combinatorial topology, and we will discuss their interests for geomeric modeling. First, two combinatorial structures (simplicial sets, combinatorial maps) are presented : we discuss the definition of data structures, basic construction operations, and we show that classical geometric construction operations can be easily defined on these structures. Topological properties can be computed on these structures, and thus, geometric computations can 
be translated into combinatorial ones. Second, complexity is discussed: it is possible, using mechanisms based upon topological properties, to reduce the complexity of data structures and algorithms, without loss of information. Remarks are mentioned about implementation, programming and geometric modelers based upon the previous notions.

I think that topological aspects are common to Geometric Modeling and Discrete Geometry (as, for instance, very natural relations exist between free-form space modeling and topology-based modeling). Some recent works establish interesting links between these fields [Fio] [Fra] [Lac] [Bru] [Som]. In the following, some of them will be mentioned, but not carefully discussed, since I will focus on methods in Topology-based Geometric Modeling.

\section{Subdivisions and combinatorial structures}

In order to illustrate basic aspects in topology-based geometric modeling, we first present two combinatorial structures: semi-simplicial sets (resp. generalized combinatorial maps, or G-maps) are used for handling simplicial subdivisions of geometric complexes (resp. cellular subdivisions of manifolds).

\subsection{Semi-simplicial sets}

An $n$-dimensional semi-simplicial set [May] is a collection of sets $K=\left(K_{k}\right)_{k=0}, \ldots n$ with maps (cf. Figure 2):

$$
\mathrm{d}_{\mathrm{i}}: \mathrm{K}_{\mathrm{k}} \rightarrow \mathrm{K}_{\mathrm{k}-1}, \mathrm{k} \geq \mathrm{l}, 0 \leq \mathrm{i} \leq \mathrm{k} \text {, }
$$

satisfying the following relations:

$$
\mathrm{d}_{\mathrm{i}} \mathrm{d}_{\mathrm{j}}=\mathrm{d}_{\mathrm{j}} \mathrm{d}_{\mathrm{i}-1}, \mathrm{j}<\mathrm{i} \text {. }
$$
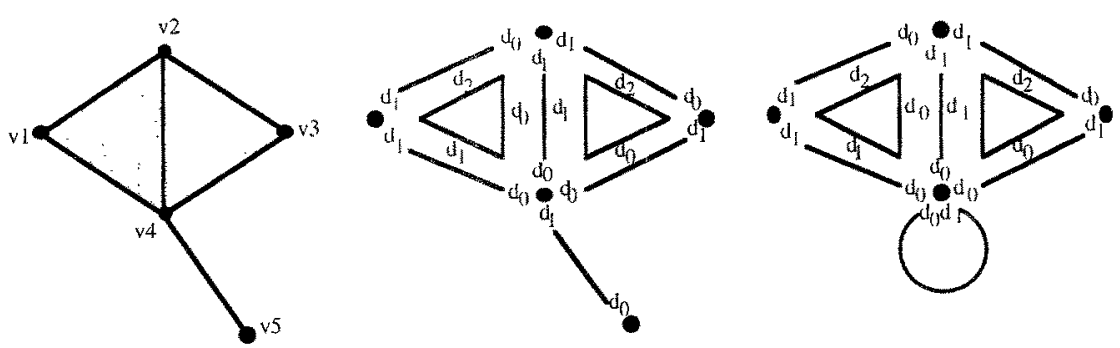

Fig. 2. 2-dimensional semi-simplicial set. The semi-simplicial set (middle) describes the topology of the triangulation (left). Each simplex correspond to a sequence of vertices; for instance, 2-simplices correspond to $(\mathrm{v} 1, \mathrm{v} 2, \mathrm{v} 4)$ and $(\mathrm{v} 2, \mathrm{v} 3, \mathrm{v} 4),(\mathrm{v} 1, \mathrm{v} 2, \mathrm{v} 4) \mathrm{d}_{0}=(\mathrm{v} 2, \mathrm{v} 4),(\mathrm{v} 1, \mathrm{v} 2, \mathrm{v} 4) \mathrm{d}_{1}=$

$(v 1, v 4),(v 1, v 2, v 4) d_{2}=(v 1, v 2)$. The semi-simplicial set (right) is constructed by identifying 0 -simplices corresponding to $v 4$ and $v 5$. Local neighbourhood of a simplex corresponds to boundary and star notions. More generally, boundary operators and their inverses make it possible to explore more or less large neighbourhoods of parts of semi-simplicial sets. 
Elements of $\mathrm{K}_{\mathrm{k}}$ are $\mathrm{k}$-dimensional abstract simplices (or $\mathrm{k}$-simplices) ; maps $\mathrm{d}_{\mathrm{i}}$ are face operators. A j-face of a $k$-simplex $(0 \leq j \leq k)$ is obtained by applying a sequence of $(k-j)$ face operators. The face is principal if the sequence is empty, otherwise it is proper. The boundary of a simplex is the set of its proper faces: for instance, $(k+1)$ face operators $\left(d_{i}\right)_{i}=0, \ldots k$ associate the $(k-1)$-simplices of its boundary with every $k$ simplex. The star of a simplex is the set of all simplices of which it is a proper face.

Any semi-simplicial set can be constructed by two operations: the creation of a $\mathrm{k}$-simplex (together with its boundary), and the identification of simplices.

The geometric realization of a semi-simplicial set is a topological space [Mil]. A topological cell, homeomorphic to the $n$-ball, is associated with any n-simplex. A $\mathrm{CW}$-complex is defined by deducing identifications of cell boundaries from face relations in the semi-simplicial sef.

\subsection{Generalized maps}

An n-dimensional generalized combinatoriall map (or n-G-map: cf. [BrSi] [Lie2] [Vin]) $\mathrm{G}$ is a set $\mathrm{D}$ with bijections (cf. Figure 3 ):

$$
\alpha_{\mathrm{i}}: \mathrm{D} \rightarrow \mathrm{D}, 0 \leq \mathrm{i} \leq \mathrm{n},
$$

such that:

$\alpha_{i}$ is an involution on $D, 0 \leq i \leq n$ (i.e. for any element $d$ of $D, d \alpha_{i} \alpha_{i}=d$ ); $\alpha_{i} \alpha_{j}$ is an involution, $0 \leq \mathrm{i}<\mathrm{i}+1<\mathrm{j} \leq \mathrm{n}$.

$D$ is a set of abstract objets called darts. A connected component of $G$ incident to dart $\mathrm{d}$ of $\mathrm{D}$ is the set of all darts that can be reached by successively applying involutions $\alpha_{i}$, starting from $d$. The $i$-cells of $G$ are defined as the connected components of the (n-1)-G-maps (D, $\alpha_{0}, \ldots, \alpha_{i}, \ldots, \alpha_{n}$ ), where $\alpha_{i}$ means that involution $\alpha_{i}$ is omitted in the sequence of involutions. If darts exist, such that they are invariant for $\alpha_{i}, G$ is with boundaries, else it is without boundaries. Boundaries of $G$ are also defined as connected components of an (n-1)-G-map.

Any $n-\mathrm{G}-\mathrm{map}$ can be constructed by two operations : the creation of a dart (which is invariant for any involution $\alpha_{\mathfrak{i}}$ ), and the sewing of two darts (cf. Figure 3).

Any n-G-map can be associated with an n-dimensional cellular quasi-manifold; conversely, any n-dimensional cellular quasi-manifold can be associated with an $n-G$ map [Lie2]. Quasi-manifolds can be with or without boundaries, orientable or not orientable. Note that it does not exist a combinatorial notion equivalent to that of manifold: quasi-manifolds make a sub-class of pseudo-manifolds; intuitively, an ndimensional cellular quasi-manifold can be constructed by sewing together $n$-cells by identifying (n-1)-cells, in such a way that at most two n-cells share an (n-1)-cell. In fact, we can prove that n-G-maps are equivalent to a sub-class of semi-simplicial sets, where simplices are structured into cells. So, notions and operations defined on semisimplicial sets can be extended on G-maps. Moreover, other notions can be defined (e.g. boundary of a G-map), since G-maps make a sub-class of simplicial quasimanifolds (i.e. semi-simplicial sets which can be constructed by creating $n$-simplices and by identifying $(n-1)$-simplices in such a way that at most two n-simplices share an ( $\mathbf{n}-1)$-simplex). 

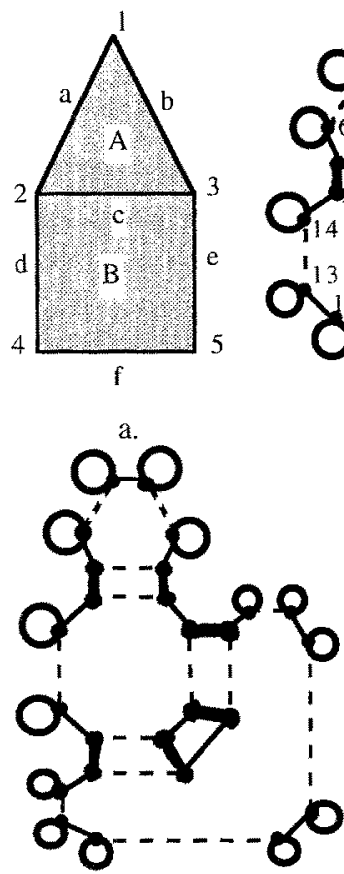

e.

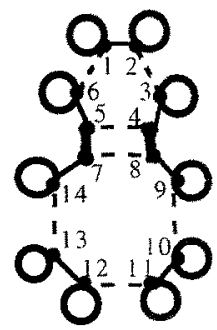

b.

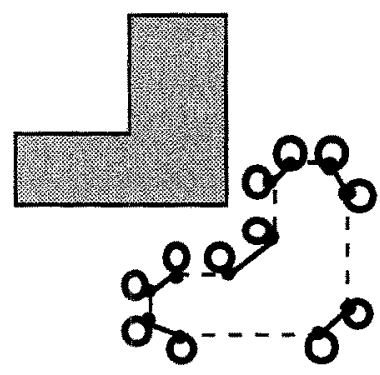

c.

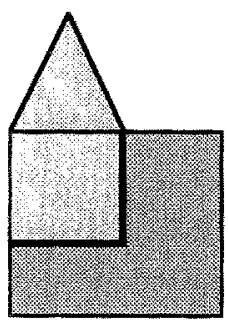

d.

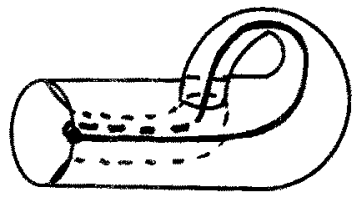

f.
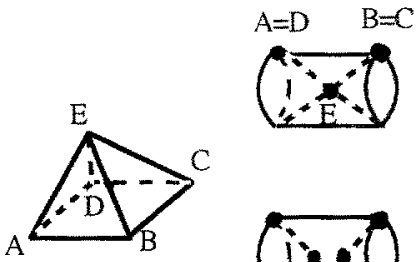

Fig. 3. a: a cellular subdivision; b: the corresponding 2-G-map: a point corresponds to a dart, involution $\alpha_{0}$ (resp. $\alpha_{1}, \alpha_{2}$ ) is symbolized by a dashed line (resp. line, thick line). All darts, except 4,5,7 and 8 , are their own images by $\alpha_{2}$. Intuitively, dart 5 (resp. $4=5 \alpha_{0}, 6=5 \alpha_{1}, 7=5 \alpha_{2}$ ) corresponds to the 3-tuple $(2, c, A)$ (resp. $(3, c, A),(2, a, A),(2, c, B)$ : cf. [Bri]).

The 2-G-map is composed by a unique connected component. Vertex 2

(resp. edge $c$, face A) corresponds to $\{6,5,7,14\}$ (resp. $\{4,5,7,8\}$, $\{1,2,3,4,5,6\})$, which darts are connected by $\alpha_{1}$ and $\alpha_{2}$ (resp. $\alpha_{0}$ and $\alpha_{2}, \alpha_{0}$ and $\left.\alpha_{1}\right)$. The 2 -G-map, and its corresponding subdivision, has one boundary, defined by $\{1,2,3,9,10,11,12,13,14,6\}$. Involutions $\alpha_{i}$ make it possible to explore the neighbourhood of any dart and cell (and also to compute properties as duality, orientability, etc.: cf. below). c: a face and its corresponding 2-G-map. d and e: identification of vertices and edges,

corresponding to sewing by $\alpha_{2}$ (contrary to semi-simplicial sets,

identification does not involve removal of elements, but only modifications of operators). $f$ : a minimal subdivision of a Klein bottle, embedded into $E^{3}$, with self-intersections (the edge is symbolized by the thick line). This is a surface subdivision which can be represented using 2 $\mathrm{G}$-maps; if we intend to model the resulting subdivision of $\mathrm{E}^{3}$, we have to explicity represent intersections and resulting volumes using 3-G-maps. g: quasi-manifolds are not manifolds. Identification of faces $(A, E, B)$ and (D,E,C) produces a quasi-manifold (up and right), which is not a manifold, 
since the neighbourhood of vertex $\mathrm{E}$ is not a ball nor a half-ball; a similar quasi-manifold can also be constructed by identifying two central points of the circular faces of a full cylinder (down right).

\subsection{Discussion}

Semi-simplicial sets are well-known objects in algebraic topology, and generalized maps can be deduced from simplicial sets (cf. section 3). Geometric objects that can be associated with these structures are well defined. Thus, numerous notions, methods and results of algebraic topology can be applied and used in geometric modeling, and experiments have shown that it is important for implementations, constructions and controls.

Semi-simplicial sets and generalized maps are combinatorial structures, from which basic data structures can be derived in a straigthforward way (for instance, simplices are implemented as records, containing pointers which correspond to boundary operators). Constraints of consistency of these data structures (thus pre and post-conditions of construction operations) are obviously deduced from the definitions of semi-simplicial sets and generalized maps [LaLi1] [BD] [Lie1].

Modeled objects are structured ones. It is thus possible to structure embedding information, and to control consistency between topology and embedding (when it is needed: cf. Figure 3.f). For instance, semi-simplicial sets linearly embedded correspond to simplicial complexes. [LaLil] has studied the modeling of semisimplicial sets embedded using triangular Bezier patches : control points are associated with simplices, in such a way that the data structure is minimal, and consistency is based upon relations deduced from boundary operators. Modelers have been designed in which generalized maps are linearly embedded [BDFL], or embedded in a hierarchical way [Bor]: hierarchized embeddings are very useful for many applications (e.g. geology [MLCF]). Complementary structures are often added when non connected objects are handled (e.g. inclusion trees [HCRR]).

Topological properties are defined on semi-simplicial sets and can be extended on generalized maps. Basic properties as incidence and adjacency relations are explicitly defined and represented in data structures, through boundary operators (in practice, inverse operators are also explicitly represented in order to get a minimal cost when accessing cell neighbours ${ }^{1}$ ). For instance for $3 \mathrm{D}$ object reconstruction from stereo images, [Som] employs a combinatorial structure for describing the topology of segmented mages, and its matching method is based upon a parallel traversal of the two structures corresponding to a pair of images (a similar structure describing the topology of the $3 \mathrm{D}$ object is constructed during this traversal). Many interesting properties can be also computed by data structure traversals. For instance, the topological surface associated with a 2-dimensional generalized map is completely defined by its number of boundaries, its orientability and its genus (using Euler characteristic) [Gri] [Tut]. which can be computed on the 2-G-map. Some properties (and algorthms for computing them) are defined for higher dimensions (e.g.

\footnotetext{
1 It is useless for involutions. since an involution is its own inverse.
} 
orientability), although there is no equivalent classification. More generally, homology groups [Ago] [Gib] can be computed on semi-simplicial sets; it is also possible to decide whether or not a semi-simplicial set belongs to a given class (homogeneous, regular, pseudo or quasi manifold, etc) by simple traversals [ElLi1].

Such topological properties are important for controlling handled objects, their constructions, for proving algorithms, etc. Since topological information is represented by combinatorial structures, these properties can be computed without numerical computing. Moreover, these properties are important in order to control computing complexity. For instance, [FBDFR] studies an algorithm for locating points in a $3 \mathrm{D}$ mesh. After numerical optimizations (and conversion of some numerical computations into combinatorial computations), this algorithm spend more than $90 \%$ of computation time in traversals; since modelled subdivisions are orientable, it is possible to employ a specialized data structure, reducing significantly the cost in space and time (cf, section 3 ).

Construction operations have been defined in algebraic topology and in geometric modeling: creating simplices or cells, identification, cartesian product (extrusion being a particular case), chamfering, cell fusion, splitting, etc. These operations are implemented into modelers, often in a straightforward way and with minimal complexity. Note that several operations are less complex on generalized maps, since some informations are implicit: cf. Figure 4. An other example is the following. Given an $n-G-m a p ~ G=\left(D, \alpha_{0}, \ldots, \alpha_{n}\right)$ which describes the topology of a cellular quasi-manifold $Q$, the dual of $G, G^{d}=\left(D, \alpha_{n}, \ldots, \alpha_{0}\right)$, describes the topology of the dual of $Q$ (i.e. a subdivision of the same space in which i-cells correspond to (n-i)cells in the initial subdivision, preserving adjacency relations).

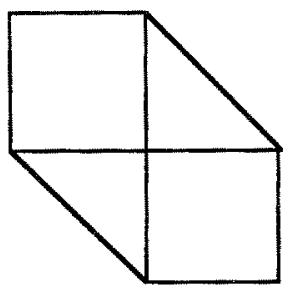

a.

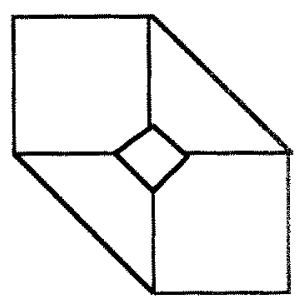

d.

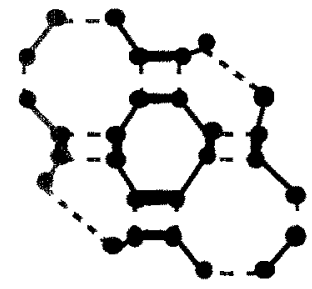

b.

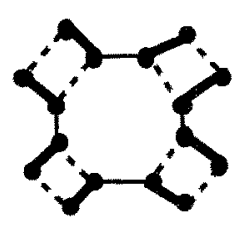

c.

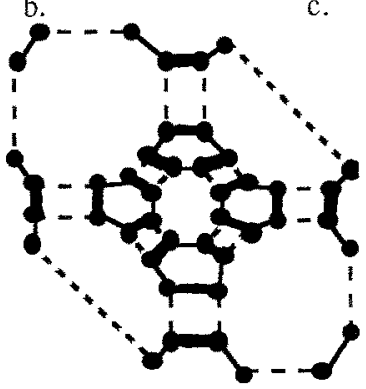

e. 


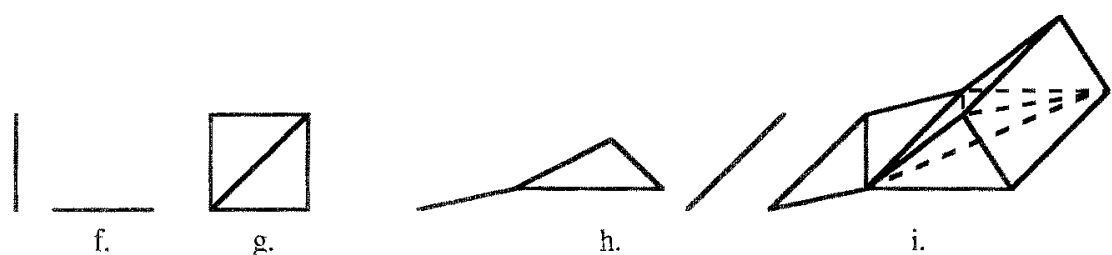

Fig. 4. a: a subdivision mainly composed by four faces; $b$. the corresponding 2-G-map (involution $\alpha_{2}$ is not represented when it is equal to identity); c. 2 "copies" of the G-map describing the central vertex; $d$. chamfering the central vertex; e. the corresponding 2-G-map, obtained by inserting $c$. (chamfering operation can be easily defined for any cell dimension and any G-map dimension). f. two edges; g the 2D semisimplicial set corresponding to their cartesian product; h: two semisimplicial set, and $\mathrm{i}$ : their 3D cartesian product.

Previous structures, notions and operations are defined for any dimension, in an homogeneous manner. So, it is often easy to extend algorithms to higher dimensions. So, generic algorithms and operations can be defined. Sometimes, generalization to higher dimensions provides simplification of algorithms. This is not always true, since some properties are not applicable, for instance:

- the Euler characteristic is defined for any semi-simplicial set (and thus for any generalized map), orientability can be computed on any generalized map, but the classification of 2-dimensional manifolds can not be generalized: cf. above.

- A local ordering of edges around vertices exists for subdivisions of surfaces. This is generalized in 3D in a local ordering of faces around edges for 3Dmanifolds, but the structure of edges around vertices is no more 1-dimensional, but 2-dimensional.

Extensibility for higher dimensions is important, since more and more applications initially concerned with (topologically) 2-dimensional problems (CAD/CAM, imagery ${ }^{2}$, etc) are now concerned with (topologically) 3D and more recently $4 \mathrm{D}$ ones (in order to take time into account).

Semi-simplicial sets (and more generally simplicial sets) are the basis of an experimental modeler [LaLi1] [LaLi2]. Abstract simplices are embedded as triangular Bezier spaces, and assemblies of such patches can thus be constructed (since a natural relation exists between indices of boundary operators and of control points). Several classical topological operations have been implemented and experimented, as identification, cut, cartesian product, and algorithms have been designed to compute homology groups of simplicial sets and their generators.

Topofil [BD] is a modeler of 3D subdivisions based upon 3-G-maps linearly embedded into $\mathrm{E}^{3}$. Classical operations in geometric modeling were adapted on 3-Gmaps, but many other operations were conceived since Topofil is employed for

2 For handling the topology of surface subdivisions, the first data structure used in topology-based modeling was conceived for computer vision problems. 
different uses and application fields, as rendering, discrete geometry (for constructing discrete objects and checking their properties), animation, medical drawings, architecture, etc. The kernel of Topofil has been used for developing modelers in geophysics, meshes, etc. With a software engineering point of view, these extensions were simplified since Topofil has been algebraically specified, increasing thus reliability and reusing $[\mathrm{BDFL}]$.

\section{Complexity}

\subsection{Reduced structures}

For many applications, very efficient data structures are needed due to data size 3 . According to the properties of the modelled subdivisions, reduced simplicial and cellular structures can be derived from semi-simplicial sets. Examples are the following (for a more complete study, see [FuLi], [ElLi1], [ElLi2]):

- An n-dimensional cubic set [Ser] is a collection of sets $\mathrm{K}=\left(\mathrm{K}_{\mathrm{m}}\right)_{\mathrm{m}=0, \ldots, \mathrm{n}}$ with operators (cf. Figure 5.a and 5.b):

$\varepsilon_{j}^{i_{j}}: K_{m} \rightarrow K_{m-1}, m \geq 1,1 \leq i \leq m, 0 \leq j \leq 1$,

satisfying the following relations:

$\varepsilon^{i} \varepsilon^{k_{1}}=\varepsilon^{k_{1}} \varepsilon^{i-1}$ with $\mathrm{i}>\mathrm{k}$.

$\mathrm{K}_{\mathrm{m}}$ is a set of m-dimensional abstract cubes. $2 \mathrm{~m}$ operators act on an $\mathrm{m}$-cube, since an $m$-cube can be defined as the cartesian product of $m$ edges, each one corresponding to a direction, and an edge has two extremities; so, $\varepsilon^{i} 0$ (resp. $\left.\varepsilon^{i}{ }_{1}\right)$ gives the (m-1)-cube at the origin (resp. extremity) along the $i^{\text {th }}$ direction. Notions and operations (similar to that related with semi-simplicial sets) can be defined on cubic sets. Semi-simplicial sets can be naturally associated with cubic sets, through cartesian product. So, for "cubic subdivisions" (i.e. subdivisions in which cells are isomorphic to cubes), the cost of cubic sets is lower than that of the associated simplicial set (cf. figure 5.c; this is similar for cubic sets and associated generalized maps).

- Simplicial quasi-manifolds make a sub-class of semi-simplicial sets (cf. section 2.2). Generalized maps correspond to cellular quasi-manifolds, which are defined as simplicial quasi-manifolds where simplices are structured into cells according to a numbering of vertices [Lie2] (this is closely related to the notion of barycentric triangulation): $\mathrm{cf}$. figure 5 ,d and 5.e;

- An n-dimensional combinatorial map (or n-map [Edm] [Jac] [Cor] [Lie1]) M is a set $D$ with bijections (cf. Figure $5 . f$ and $5 . \mathrm{g}$ ):

$\beta_{\mathrm{i}}: \mathrm{D} \rightarrow \mathrm{D}, 1 \leq \mathrm{i} \leq \mathrm{n}$,

such that:

$\beta_{1}$ is a permutation on $D, \beta_{i}$ is an involution on $D, 2 \leq i \leq n$;

$\beta_{i} \beta_{j}$ is an involution, $1 \leq i<i+1<j \leq n$.

${ }^{3}$ See also the study in $[\mathrm{BF}][\mathrm{FB}]$ about the complexity of $2 \mathrm{D}$ and $3 \mathrm{D}$ subdivisions. 
Given a connected orientable n-G-map without boundaries $G=\left(D, \alpha_{0}, \ldots, \alpha_{n}\right)$, two n-maps $M_{1}$ and $M_{2}$ can be associated with $G$, each one corresponding to a possible orientation of $G$, i.e. : let $d$ be a dart of $D ; M_{1}=\left(D_{1}, \beta_{1}, \ldots, \beta_{n}\right)$ where $\beta_{i}=\alpha_{0} \alpha_{i} / D_{1}, 1 \leq i \leq n$, and $D_{1}$ is the set of all darts which can be reached starting from $d$ by successively applying $\beta_{i}(1 \leq i \leq n)$ (the definition of $M_{2}$ is similar, and $d \alpha_{0}$ belongs to $M_{2}$ ). In practice, data structures deduced from $\mathrm{n}$ maps are used for handling orientable cellular quasi-manifolds, with a lower space complexity than n-G-maps, since one dart of an n-map corresponds to two darts of the associated n-G-map.
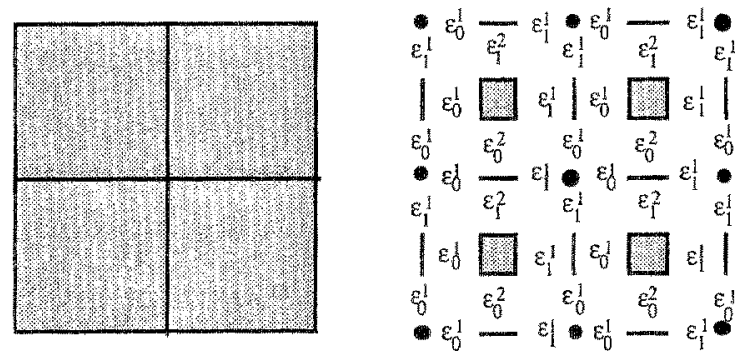

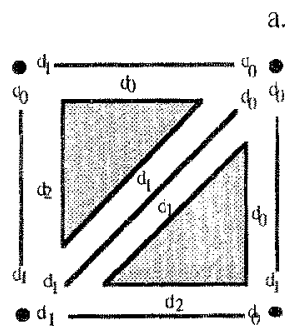

c.

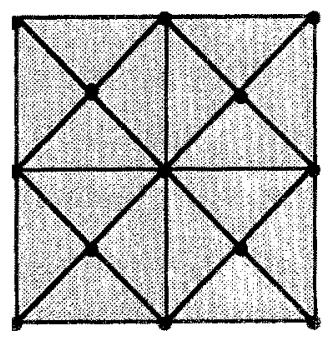

f.

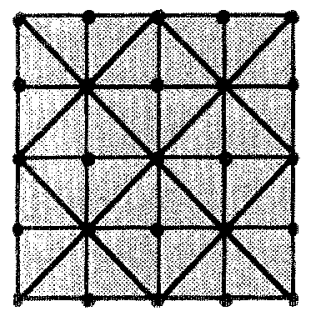

d.

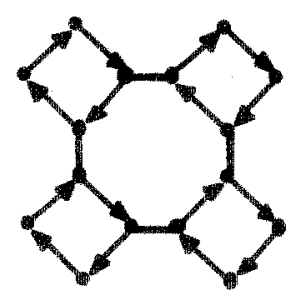

g.
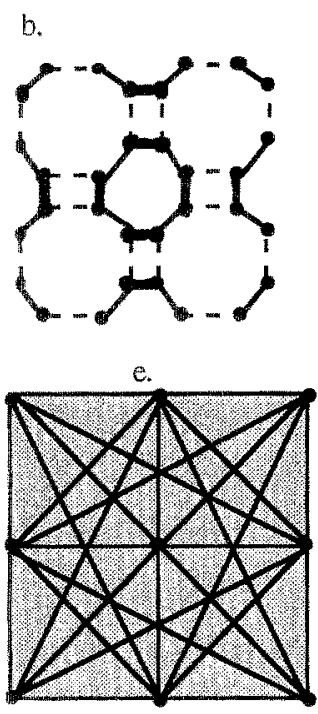

h.

Fig. 5. a: a cubic subdivision, b: a corresponding cubic set. $\mathrm{c}$ : a semisimplicial set associated with a 2 -cube of $b$. d. the barycentric triangulation of a; each cell is split by inserting a vertex at its barycenter, numbered with the cell dimension. e: the corresponding 2-G-map (involution $\alpha_{2}$ is not represented when it is equal to identity); a dart is associated with each simplex, and involutions are deduced from boundary operators and vertex numbering. $f$; since the subdivision of $d$. is orientable, simplices can be merged two by two. g. the corresponding 2-map. which defines an orientation of $\mathrm{d}$. $\mathrm{h}$. a subdivision of a. where cells are not regular. 
Other structures can be deduced from sub-classes of semi-simplicial sets (even cellular structures, defined as structured semi-simplicial sets), according to some mechanisms which employ the properties of sub-classes in order to reduce the information explicitly represented. e.g.:

- when cells are cartesian products of simplices, the corresponding triangulation of cells is not explicitly represented in cubic or simploidal sets [FuLi];

- chains [EILi2] are deduced from semi-simplicial sets in which simplices are structured into cells (chains are associated with cellular complexes) and G-maps can be deduced from chains representing cellular manifolds (it is useless to explicitly represent $0-, \ldots,(\mathrm{n}-1)$-cells, and the related boundary operators, when handling manifolds; this is also true for simplicial manifolds).

Other mechanisms exist for other properties [EILil]. Main interest here consists in reducing the space complexity of data structures (information becomes implicit), and advantages presented before remain, since conversions between structures do not involve loss of information. For instance, for some very regular subdivisions as presented in figure 5, the explicit representation of all topological information is useless for many algorithms (even cubic sets are not optimal). Simplicial sets, cubic sets, etc., are needed in order to handle irregular assemblies of regular cells. Chains, generalized maps, etc. make it possible to handle irregular assemblies of irregular cells.

Operations for computing topological properties and construction operations have to be adapted to reduced structures. Time complexity of these operations can thus be strongly reduced (for instance, time complexity of some modification operations is reduced since less information has to be explicitly modified, for instance sewing or chamfering G-maps) or increased (when it is necessary to explicitly compute information being implicit in the handled structure). No general results exist, and it is necessary to carefully analyse space and time complexity according to the type of objects and operations which are needed for a particular use (partial results are presented in [EILi1]).

In order to handle complex assemblies of free-form spaces, a modeler of simploids has been developed [FuLi], since natural links which exist between simplicial sets and control points of free-form spaces can be extended for simploidal sets and general simplicial algorithms.

[BeLi] studies the conception of Multifil, a modeler of cellular and simplicial complexes based upon the hierarchy of structures presented above, for handling general complexes with a minimal complexity in space and time. Hybrid structures are also studied, since few singularities exist in objects for many applications. For instance, usual objects are manifolds except in some few parts; it is thus useless to employ a general structure for representing all parts of the object: a manifold structure can be used, enriched when it is necessary to represent "non manifold" parts (similar mechanisms are used in [Mar]). 


\subsection{Hierarchized embeddings}

For some applications, objects are structured into different levels, for representing informations related to the objects (for instance, a geographic map) or for optimizing time or space complexity (for instance different resolution levels can be distinguished in order to decrease time complexity of algorithms). Several approaches are based upon the natural recursivity of the definition of embedding [Bor] (a cell can be embedded onto a part of geometric space which is itself subdivided).

The key point here is consistency between topology and embedding. Since a discrete (or hierarchized) embedding is a "combinatorial embedding", its properties can be exactly defined and preserved through constructions. For instance, combinatorial maps are employed by [BrGu] [Bru] in order to handle segmented images: a 2dimensional map is embedded into $Z^{2}$ by associating an "inter-pixel" path with each edge; these paths are the frontiers of regions, associated with faces, and their intersections are associated with vertices. For reconstructing 3D objects from stereo images, [Som] uses two 2-dimensional maps in order to describe the topology of a pair of segmented images, and an other 2-map describes the topology of the 3D object. This map is embedded into $\mathrm{E}^{3}$, but also into the two other maps in order to control consistency between the object and its images (and to control its construction).

Problems related to consistency in a multi-level hierarchized embedding were studied by [Fey]. He proposed a definition of embedding of simplicial (and cellular) structures into lower-level simplicial (resp. cellular) structures (the lowest level being a regular subdivision of the 2D and 3D space, vertices being embedded onto points of $Z^{2}$ and $Z^{3}$ ), and of some properties of embedding which are necessary for applying some operations (e.g. boolean operations), in such a way that their results can be propagated to higher or lower levels.

\section{Conclusion}

Numerous combinatorial structures have been defined for representing regular or irregular subdivisions of geometric objects, for any dimension, and many construction operations and operations for computing topological properties have been proposed and experimented.

Using these structures, it is possible to handle "discretizations" of geometric objects, since they define the topology of structured continuous or discrete objects. Since they are combinatorial ones, data structures and algorithms have been defined without any loss of properties, and several modelers have been designed for different application fields as CAD, animation and image synthesis, architecture, digital imagery, etc.

Among numerous research directions, I think a main one is the following: Several works have shown that some results of topology-based geometric modeling 
can be useful for Discrete Geometry and Computer Imagery. A careful study of the usefulness of structures and algorithms has to be made, i.e. I think it is important to systematically study classical algorithms in Discrete Geometry and Imagery in order to get a definitive conclusion about the possible interests of Topology-based Modeling methods for these fields (thus following approaches of [Fio], [Lac], etc.). A step could be the conception of a modeler of discrete objects. It is thus important to carry on investigating algebraic topology in order to select and experiment constructions and properties which can be useful for geometric modelers and algorithms in Discrete Geometry.

\section{Acknowledgements}

I wish to thank (ex-)members of the Computer Science Research Center at Strasbourg, mainly J. Françon and Y.P. Réveilles, Y. Bertrand, P. Borianne, H. Elter, C. Fey, L. Fuchs and V. Lang.

\section{References}

[Ago] Agoston, M.: Algebraic Topology: A First Course. Pure and Applied Mathematics, Marcel Dekker Ed., New-York, U.S.A. (1976)

[AFF] Ansaldi, S., de Floriani, L., Falcidieno, B.: Geometric Modeling of Solid Objects by Using a Face Adjacency Graph Representation. Computer Graphics 19,3 (1985) 131-139

[Ale] Alexandroff: Combinatorial Topology. Graylock Press, Rochester, New-York, USA (1957)

[ArKo] Arquès, D., Koch, P.: Modélisation de solides par les pavages. Proc. of Pixim'89, Paris, France (1989) 47-61

[Bau] Baumgart, B.: A Polyhedron Representation for Computer Vision. AFIPS Nat. Conf. Proc. 44 (1975) 589-596

[BD] Bertrand, Y., Dufourd, J.-F.: Algebraic specification of a 3D-modeler based on hypermaps. CGVIP, 1 (1994) 29-60

[BDFL] Bertrand, Y., Dufourd, J.-F., Françon, J., Lienhardt, P.: Algebraic Specification and Development in Geometric Modeling. Proc. of TAPSOFT93 Orsay, France (1993)

[BeLi] Bertrand, Y., Lienhardt, P.: Spécification algébrique et sortes ordonnées pour un multi-modeleur d'extensions des cartes. Journées du GDR/PRC de Programmation, Orléans, France (1996).

[BF] Bertrand, Y., Françon, J.: 2D manifolds for Boundary Representation: A statistical study of the cells. Research Report R97 8, Université Louis Pasteur, Strasbourg, France (1997)

[Bor] Borianne, P.: Conception of a Modeller of Subdivisions of Surfaces based on Generalized Maps. PhD Thesis 037, Université Louis Pasteur, Strasbourg, France (1991)

[Bra] Braid, I .: The Synthesis of Solids Bounded by Many Faces. Communications of the A.C.M. 18,4 (1975) 209-216 
[BrGu] Braquelaire. J.-P., Guitton. P.: A Model for Image Structuration. Proc. of Computer Graphics International'88 Genève. Switzerland (1988)

[Bri] Brisson, E.: Representing Geometric Structures in D Dimensions: Topology and Order. Discrete and Computational Geometry 9 (1993) 387-426

[Bru] Brun, L.: Segmentation d'images à base topologique. PhD Thesis 1651, Université de Bordeaux I, Bordeaux, France (1996)

[BrSi] Bryant, R., Singerman, D.: Foundations of the Theory of Maps on Surfaces with Boundaries. Quart. Journal of Math. Oxford 2,36 (1985) 17-41

[CCM] Cavalcanti, P.R.. Carvalho, P.C.P., Martha, L.F.: Non-manifold modeling: an approach based on spatial subdivision. Computer-Aided Design 29,3 (1997) 299-220

[Cor] Cori, R. Un code pour les graphes planaires et ses applications. Astérisque 27 (1975)

[CrRe] Crocker, G., Reinke, W.: An Editable Non-Manifold Boundary Representation. Computer Graphics and Applications 11,2 (1991)

[DoLa] Dobkin, D., Laszlo, M.: Primitives for the Manipulation of Three-Dimensional Subdivisions. Proc. of $3^{\text {rd }}$ Symposium on Computational Geometry Waterloo, Canada (1987) $86-99$

[Edm] Edmonds, J.: A Combinatorial Representation for Polyhedral Surfaces. Notices Amer. Math. Soc. 7 (1960)

[ElLi1] Elter, H., Lienhardt. P.: Different Combinatorial Models based on the Map Concept for the Representation of Different Types of Cellular Complexes. IFIP TC5/WG5.10 Working Conterence on Geometric Modeling in Computer Graphics, Genova. Italy (1993) [EiLi2] Elter, H., Lienhardt, P.: Cellular complexes as structured semi-simplicial sets. Int. Journal of Shape Modeling. 1.2 (1994) 191-217

[FB] Françon, J., Bertrand, Y.: 3D manifolds for Boundary Representation: A statistical study of the cells. Research Report R96-9, Universitế Louis Pasteur, Strasbourg. France (1997)

[FePa] Ferruci, V., Paoluzzi, A.: Extrusion and Boundary Evaluation for Multidimensional Polyhedra. Computer-Aided Design 23,1 (1991) 40-50

[Fey] Fey, C.: Etude de plongements hiérarchisés de subdivisions simpliciales ou cellulaires, PhD Thesis, Université Louis Pasteur, Strasbourg, France (1996)

[Fio] Fiorio, C: A Topologically Consistent Representation for Image Analysis: The Frontiers Topological Graph. Proc. of DGCl96, Lyon. France (1996) 151-162

[FBDFR] Fousse. A., Bertrand, Y., Dufourd, J.-F., Françon, J., Rodriguès, D.: Localisation des points d'un maillage généré en yue de calculs en différences finies. Journées "Modélisation du sous-sol", Orléans, France (1997).

[Fra] Françon, J.: On Recent Trends in Discrete Geometry in Computer Science. Proc. DGCI'96, Lyon, France (1996) 3-16

[FrPi] Fritsch, R., Piccinini, R. A.: Cellular Structures in Topology. Cambridge University Press (1990)

[FuLi] Fuchs, L.. Lienhardt, P.: Topological Structures for d-Dimensional Free-Form Objects. CAGD'97. Lillehammer. Norway (1997)

[GHPT] Gangnet, M., Hervé. J.-C.. Pudet, T., Van Thong, J.-M.: Incremental Computation of Planar Maps. Computer Graphics 23,3 (1989) 345-354

[Gib] Giblin. P.J.: Graphs, surfaces and homology. Chapman and Hall, London, UK (1977) 
[Gri] Griffiths, H.-B. Surfaces. Cambridge University Press Cambridge. U.K. (1981)

[GuSt] Guibas, L., Stolfi, J.: Primitives for the Manipulation of General Subdivisions and the Computation of Voronoï Diagrams. A.C.M. Transactions on Graphics 4,2 (1985) 74123

[GCP] Gursoz, E. L.. Choi, Y., Prinz. F. B.: Vertex-based Representation of NonManifolds Boundaries. in Geometric Modeling for Product Engineering M. Wozny, J. Turner and K. Preiss eds.. North-Holland (1989) 107-130

[HC] Hansen, O.H., Christensen, N.J.: A model for n-dimensional boundary topology. Proc. 2nd ACM Symp. Solid Modeling Foundations and CAD/CAM Applications (J. Rossignac and J. Turner eds) Montréal. Canada (1993) 65-73

[HCRR] Halbwachs, Y.. Courrioux. G., Renaud, X., Repusseau, P.: Topological and Geometric Characterization of Fault Networks Using 3-Dimensional Generalized Maps. Mathematical Geology, 28.5 (1996) 625-656

[Jac] Jacque, A.: Constellations et Graphes Topologiques. Colloque Math. Soc. Janos Bolyai (1970) 657-672

[KIE] Kenmochi, Y., Imiya, A., Ezquerra, N.: Polyhedra generation from lattice points. Proc. DGCI'96. Lyon. France (1996) $127-138$

[Lac] Lachaud, J.-O.: Topologically Defined Isosurfaces. Proc. of DGCI'96, Lyon, France (1996) 245-256

[LaLi1] Lang, V., Lienhardt, P.: Geometric Modeling with Simplicial Sets. Proc. of CGI'96, Pohang, Korea (1996)

[LaLi2] Lang, V., Lienhardt, P.: Cartesian Product of Simplicial Sets. Proc. of WSCG'97, Plzen, Czech Republic (1997)

[Lie1] Lienhardt. P.: Topological Models for Boundary Representation: a Comparison with N-Dimensional Generalized Maps. Computer-Aided Design 23,1 (1991) 59-82

[Lie2] Lienhardt, P.: N-Dimensional Generalized Combinatorial Maps and Cellular QuasiManifolds. Int. Journal of Computational Geometry and Applications 4,3 (1994) 275324

[LuLu] Luo, Y.. Lukacs. G. A.: A Boundary Representation for Form-Features and NonManifold Solid Objects. Proc. of I $^{\text {st }}$ ACM/Siggraph Symposium on Solid Modeling Foundations and CAD/CAM Applications Austin, Texas, U.S.A. (1990)

[Män] Mäntylä, M.: An Introduction to Solid Modeling. Computer Science Press Rockville, U.S.A. (1988)

[May] May, J. P.: Simplicial Objects in Algebraic Topology. Van Nostrand Princeton (1967)

[Mar] Marcheix, D.: Modélisation géométrique d'objets non-variétés: construction, représentation and manipulation. PhD Thesis, Université de Bordeaux I, Bordeaux, France (1996)

[Mil] Minnor: The geometric realization of semi-simplicial complexes. Ann. of Math. 65 (1957) 357-362

[MLCF] Mallet, J.-L., Levy. B., Conreaux, S., Fousse, A.: G-maps. a new topological model for gOcad. 15th Gocad Meeting. Vancy, France (1997)

[MuHi] Murabata, S.. Higashi, M.: Non-manifold geometric modeling for set operations and surface operations. IFIP/RPI Geometric Modeling Conference Rensselaerville, N.Y. (1990) 
[PBCF] Paoluzzi, A., Bernardini, F., Cattani, C., Ferrucci, V.: Dimension Independent Modeling with Simplicial Complexes. ACM Transactions on Graphics, 12, 1 (1993)

[RoOC] Rossignac, J.. O'Connor, M.: SGC: A Dimension-Independent Model for Pointsets with Internal Structures and Incomplete Boundaries. in Geometric Modeling for Product Engineering M. Wozny, J. Tumer and K. Preiss eds., North-Holland (1989) 145-180

[Ser] Serre,J.-P.: Homologie singulière des espaces fibrés. Ann. of Math. 54 (1951) 425505

[SeTh] Seifert, H., Threlfall, W.: A textbook of topology. Academic Press New York, (1980)

[Som] Sommellier, L.: Mise en correspondance d'images stéréoscopiques utilisant un modèle topologique. PhD Thesis. Université Claude Bernard, Lyon, France (1997)

[Spe] Spehner, J.-C.: Merging in Maps and Pavings. Theoretical Computer Science 86, (1991) 205-232

[Tak] Takala, T.: A taxonomy of geometric and topological models. Computer Graphics and Mathematics (B. Falcidieno. I. Herman and C. Pienovi eds.), Springer (1992)

[Tut] Tutte, W. Graph Theory. Encyclopaedia of Mathematics and its Applications, Addison Wesley, Menlo Park, U.S.A. (1984)

[Vin] Vince, A.: Combinatorial Maps. Joumal of Combinatorial Theory Series B 34 (1983) $1-21$

[Weil] Weiler, K.: Edge-based Data Structures for Solid Modeling in Curved-Surface Environments. Computer Graphics and Applications 5,1 (1985) 21-40

[Wei2] Weiler, K.: The Radial-Edge Data Structure: A Topological Representation for NonManifold Geometry Boundary Modeling. Proc. IFIP WG 5.2 Working Conference Rensselaerville, U.S.A. (1986), in Geometric Modeling for CAD Applications Elsevier (1988) $3-36$ 